\title{
Voltage Gain-Controlled Third-Generation Current Conveyor and its All-Pass Filter Verification
}

HERENCSÁR, N.; KARTCI, A.; KOTON, J.; TSIRIMOKOU, G.; PSYCHALINOS, C.

Proceedings of the 201723 European Conference on Circuit Theory and Design (ECCTD 2017)

pp. 1-4

elSBN: 978-1-5386-3974-0

DOI: http://dx.doi.org/10.1109/ECCTD.2017.8093273

Accepted manuscript

(C)2017 IEEE. Personal use of this material is permitted. Permission from IEEE must be obtained for all other uses, in any current or future media, including reprinting/republishing this material for advertising or promotional purposes, creating new collective works, for resale or redistribution to servers or lists, or reuse of any copyrighted component of this work in other works. Norbert Herencsar, Aslihan Kartci, Jaroslav Koton, Georgia Tsirimokou, Costas Psychalinos, "Voltage Gain-Controlled Third-Generation Current Conveyor and its All-Pass Filter Verification", Proceedings of the 201723 European Conference on Circuit Theory and Design (ECCTD 2017), pp. 1-4, 2017. DOI: 10.1109/ECCTD.2017.8093273. Final version is available at http://ieeexplore.iee.org/document/8093273/ 


\section{Voltage Gain-Controlled Third-Generation Current Conveyor and its All-Pass Filter Verification}

\author{
Norbert Herencsar ${ }^{1}$, Aslihan Kartci ${ }^{1,2}$, Jaroslav Koton ${ }^{1}$ \\ ${ }^{1}$ Dept. of Telecommunications / ${ }^{2}$ Dept. of Radio Electronics \\ Brno University of Technology \\ Technicka 3082/12, 61600 Brno, Czech Republic \\ \{herencsn; kartci; koton\}@feec.vutbr.cz
}

\author{
Georgia Tsirimokou, Costas Psychalinos \\ Physics Department, Electronics Laboratory \\ University of Patras \\ Rio Patras, GR-26504, Greece \\ tsirimg@upatras.gr; cpsychal@physics.upatras.gr
}

\begin{abstract}
The paper presents a new active building block (ABB) called minus-type voltage gain-controlled third-generation current conveyor (VGC-CCIII-) in which the voltage gain between $Y$ to $X$ terminal can be controlled. The usefulness of the tunable feature in the presented $\mathrm{ABB}$ is demonstrated in currentmode $\left\{0.3^{\text {rd }} ; 0.5^{\text {th }} ; 0.7^{\text {th }} ; 1^{\text {st }}\right\}$-order all-pass filter (APF) employing single VGC-CCIII-, one capacitor, and one resistor. The theoretical results of the integer- and fractional-order APF are verified by SPICE simulations based on readily available IC OPA860 macromodel, which can also be used in experiments.
\end{abstract}

Keywords-all-pass filter; current-mode; fractional-order filter; voltage gain-controlled third-generation current conveyor

\section{INTRODUCTION}

Although the very first notice about the third-generation current conveyor (CCIII) has appeared in the technical literature in 1982 [1], its first implementations have been presented much later, in 1995 [2], [3]. Since then versatility of the CCIII was demonstrated in various applications such as current-mode (CM) biquadratic filter design with minimal configuration, higher-order immittance function synthesis, synthesis of R-L and C-D immittances, actively simulated grounded lossy inductors, or first-order all-pass filters (APF) [4]-[9]. Later the third-generation controlled conveyor (CCCIII) was also introduced for floating resistance realization in which the intrinsic resistance of the input $\mathrm{X}$ terminal can be controlled by DC bias current [10]. For easy tunability of the circuit parameters by either voltage or current and in order to increase the versatility of conventional active building blocks (ABBs), the electronically-tunable secondgeneration current conveyor (ECCII) [11], programmable current amplifier (PCA) [12], the voltage and current gain CCII (VCG-CCII) [13], and the controlled gain current inverter differential output buffered amplifier (CG-CIBA) [14] were introduced. This work aims to introduce a new tunable ABB called minus-type voltage gain-controlled thirdgeneration current conveyor (VGC-CCIII-). The macromodel of the readily available IC OPA860 [15] has been used for the implementation of VGC-CCIII- in which the variable voltage gain $h$ between $\mathrm{Y}$ and $\mathrm{X}$ terminals can be set by ratio of two grounded resistors. The usefulness of the introduced VGCCCIII- is demonstrated on tunable CM APF of orders $\alpha \in\{0.3 ; 0.5 ; 0.7 ; 1\}$. Numerous SPICE simulation results are included to support the theory.

This article is based upon work from COST Action CA15225, a network supported by COST (European Cooperation in Science and Technology). Research described in this paper was financed by the National Sustainability Program under grant LO1401 and by the Czech Science Foundation under grant no. 16-11460Y. For the research, infrastructure of the SIX Center was used.

\section{CIRCUIT DESCRIPTION}

\section{A. Minus-Type Voltage Gain-Controlled Third-Generation Current Conveyor (VGC-CCIII-)}

The minus-type voltage gain-controlled third-generation current conveyor (VGC-CCIII-) is a three-terminal device and its circuit symbol is shown in Fig. 1. Compared to the conventional CCIII its voltage transfer from $\mathrm{Y}$ to $\mathrm{X}$ terminal can be tuned by means of the voltage gain $h$. The relations between the individual terminals of the VGC-CCIII- can be described by the following equations:

$$
i_{Y}=-\gamma i_{X}, \quad v_{X}=h \cdot \beta v_{Y}, \quad i_{Z-}=-\eta i_{X}
$$

where $\gamma=1-\varepsilon_{i}, \quad \beta=1-\varepsilon_{v}$, and $\eta=1-\varepsilon_{j}$. Here, $\varepsilon_{i}, \quad \varepsilon_{j}$ $\left(\left|\varepsilon_{i}\right|,\left|\varepsilon_{j}\right| \ll 1\right)$ denote current tracking errors and $\varepsilon_{v} \quad\left(\left|\varepsilon_{v}\right| \ll 1\right)$ denotes voltage tracking error of VGC-CCIII-, respectively, and the $h$ represents the voltage gain.

For the purpose of simulations, CMOS or BJT transistor internal implementation of active elements is commonly used [2]-[8]. However, in these internal implementations the transistor models' level are not clearly specified. Hence, the evaluation of the real behavior of the analyzed functional block is not fully credible. For this reason, more often the simulations are done using readily available active elements that are suitably interconnected. Here, we can mention the OPA860 [15] that contains the so-called 'diamond' transistor (DT) and fast voltage buffer (VB), and that was used e.g. in [16] to design the conventional CCIII. In Figs. 2(a) and (b) the schematic symbols of DT and VB including the main parasitic elements are shown. According [15] these parasitics can be evaluated as: $R_{b}=455 \mathrm{k} \Omega, C_{b}=2.1 \mathrm{pF}, R_{c}=54 \mathrm{k} \Omega, C_{c}=2 \mathrm{pF}$, $R_{e}=10.5 \Omega, R_{V B i}=1 \mathrm{M} \Omega, \quad C_{V B i}=2.1 \mathrm{pF}, \quad$ and $\quad R_{V B o}=1 \Omega$. Using the OPA860, the implementation of the proposed

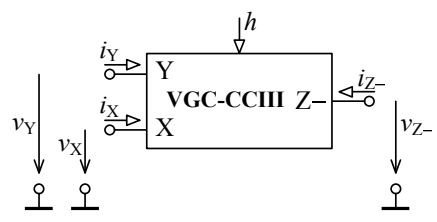

Fig. 1. Circuit symbol of VGC-CCIII-. 


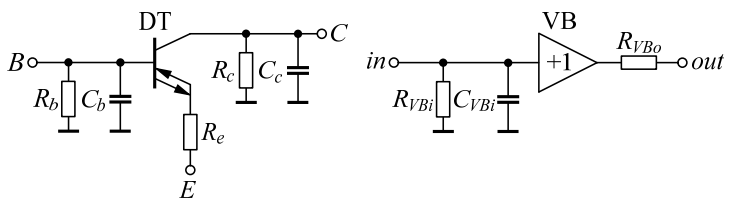

(a)

(b)

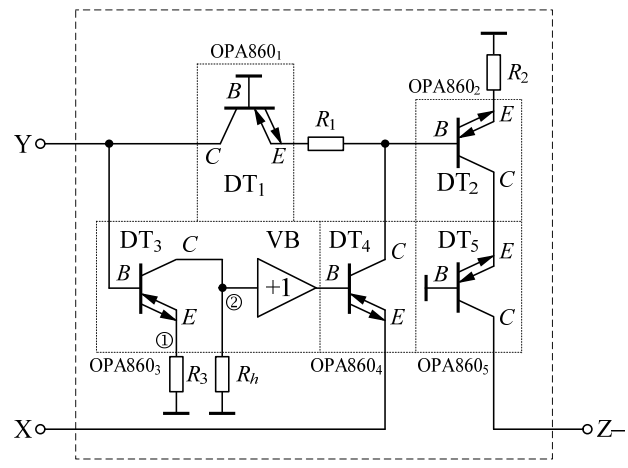

(c)

Fig. 2. (a) 'Diamond' transistor (DT), (b) voltage buffer (VB) including main parasitics, (c) VGC-CCIII- implementation by OPA860 ICs.

VGC-CCIII- is given in Fig. 2(c), where only the external resistors setting the voltage gain $h$ are given without showing the parasitics of its sub-circuits. The variable voltage gain $h$ between $\mathrm{Y}$ and $\mathrm{X}$ terminals can be set by ratio of the grounded resistors $R_{h}$ and $R_{3}$, where the ideal voltage gain is $h_{i d}=R_{h} / R_{3}$. However, taking into account parasitic resistances of $\mathrm{DT}_{3}$ and VB at nodes (1) and (2), i.e. $R_{e}$, which appears in series with $R_{3}$ and resistances $R_{c}, R_{V B i}$ that appears in parallel with $R_{h}$, the voltage gain $h$ converts to $h=R_{h}\left\|R_{c}\right\| R_{V B i} /\left(R_{3}+R_{e}\right)$. Moreover note that the current transfer between $\mathrm{X}$ and $\mathrm{Z}$ terminals is theoretically unity under condition $R_{1}=R_{2}$.

\section{B. Integer-Order All-Pass Filter Design}

The usefulness of the introduced ABB is demonstrated on tunable CM first-(integer)-order APF adopted from [8], where this circuit uses conventional CCIII. First-order APF is often used in analog signal processing in order to shift the phase of an electrical signal between $180^{\circ}$ (at $\omega=0$ ) to $0^{\circ}($ at $\omega=\infty$ ) and $0^{\circ}($ at $\omega=0)$ to $-180^{\circ}$ (at $\omega=\infty$ ) while keeping the amplitude constant [8], [9], [17], [18]. The tunable CM APF using canonic number of passive and active elements is shown in Fig. 3 [8]. Considering the ideal VGC-CCIII- (i.e. $\gamma, \beta$, and $\eta$ being unity), routine analysis yields current transfer function (TF) and the phase of the filter in following form:

$$
T(s)=\frac{I_{\text {out }}}{I_{\text {in }}}=\frac{1-s C R / h}{1+s C R / h}, \quad \varphi(\omega)=-2 \tan ^{-1}\left(\frac{\omega C R}{h}\right) .
$$

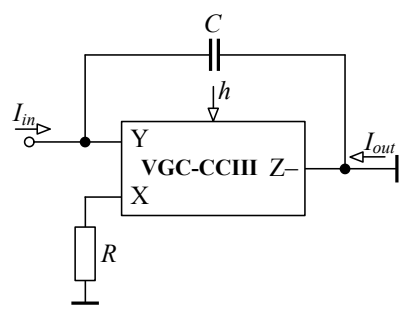

Fig. 3. Tunable current-mode all-pass filter adopted from [8].
From Eq. (2a) the zero $\left(\omega_{z}\right)$ and pole $\left(\omega_{p}\right)$ frequencies can be expressed as $\omega_{z}=\omega_{p}=h / C R$, while from Eq. (2b) it can be seen that the AFP provides phase shifting between $0^{\circ}$ to $-180^{\circ}$. Taking into account the non-ideal current and voltage gains of the VGC-CCIII-, TF in Eq. (2a) converts to:

$$
T^{\prime}(s)=\frac{I_{\text {out }}}{I_{\text {in }}}=\frac{1-s C R /(h \cdot \beta \eta)}{1+s C R /(h \cdot \gamma \beta)},
$$

and the non-ideal phase response from Eq. (3) is given as:

$$
\varphi^{\prime}(\omega)=-\tan ^{-1}\left(\frac{\omega C R}{h \cdot \beta \eta}\right)-\tan ^{-1}\left(\frac{\omega C R}{h \cdot \gamma \beta}\right) .
$$

From Eqs. (3) and (4) it can be seen that the non-idealities of the VGC-CCIII- slightly affect the magnitude and phase responses of the filter. Consequently, the non-ideal $\omega_{z}^{\prime}$ and $\omega_{p}^{\prime}$ frequencies of the filter can be found as $\omega_{z}^{\prime}=\beta \eta \cdot h / C R$, $\omega_{p}^{\prime}=\gamma \beta \cdot h / C R$, and the active and passive sensitivities of both $\omega_{z}^{\prime}$ and $\omega_{p}^{\prime}$ are given as $S_{\beta, \eta, h}^{\omega_{z}^{\prime}}=-S_{C, R}^{\omega_{z}^{\prime}}=S_{\gamma, \beta, h}^{\omega_{p}^{\prime}}=-S_{C, R}^{\omega_{p}^{\prime}}=1$ from which it is evident that all sensitivities of active parameters and passive components for both $\omega_{z}^{\prime}$ and $\omega_{p}^{\prime}$ are unity in relative amplitude. Hence, the proposed filter shows low sensitivity performance.

\section{Fractional-Order All-Pass Filter Design}

Recently, the fractional-(non-integer)-order continuoustime systems were labeled as the systems of $21^{\text {st }}$ century due to the interdisciplinary nature of fractional calculus [19]. During the last two decades the application areas of fractional calculus such as modeling of biological tissues, control, electrochemistry, electromagnetism, diffusion theory, and/or internet traffic have significantly increased [19]. Fractionalorder capacitors with pseudo-capacitance $C_{\alpha}(0<\alpha<1)$ of impedance $Z_{C \alpha}(s)=1 / C_{\alpha} s^{\alpha}$ are important components for realizing fractional-order circuits [20]. Hence, considering the proposed APF with replacement of capacitor $C$ of $\alpha=1$ with fractional-order one (unit $\mathrm{F} / \mathrm{s}^{1-\alpha}[21]$ ), the TF in (2a) turns to:

$$
T_{\alpha}(s)=\frac{I_{\text {out }}}{I_{\text {in }}}=\frac{1-s^{\alpha} C_{\alpha} R / h}{1+s^{\alpha} C_{\alpha} R / h} .
$$

Now, to evaluate the magnitude, phase, and subsequently pole frequency responses, the $s^{\alpha}$ is replaced by $\omega^{\alpha}[\cos (\alpha \pi / 2)+j \cdot \sin (\alpha \pi / 2)]$ [22]. Hence, the corresponding expressions are:

$$
\begin{aligned}
& \left|T_{\alpha}(\omega)\right|=\frac{I_{\text {out }}}{I_{\text {in }}}=\sqrt{\frac{1-2 \omega^{\alpha}\left(C_{\alpha} R / h\right) \cos (\alpha \pi / 2)+\omega^{2 \alpha}\left(C_{\alpha} R / h\right)^{2}}{1+2 \omega^{\alpha}\left(C_{\alpha} R / h\right) \cos (\alpha \pi / 2)+\omega^{2 \alpha}\left(C_{\alpha} R / h\right)^{2}},} \\
& \varphi_{\alpha}(\omega)=-\tan ^{-1}\left\{2 \omega^{\alpha}\left(C_{\alpha} R / h\right) \sin (\alpha \pi / 2) /\left[1-\omega^{2 \alpha}\left(C_{\alpha} R / h\right)^{2}\right]\right\}, \\
& \omega_{\alpha p}=\left\{\omega_{\alpha z}-2 h \cdot[\cos (\alpha \pi / 2)] / C_{\alpha} R\right\}^{1 / \alpha}, \\
& (6 \mathrm{a}, \mathrm{b}, \mathrm{c})
\end{aligned}
$$

in which the zero frequency expression is:

$$
\omega_{\alpha z}=h\left[\cos (\alpha \pi / 2)-\sqrt{\cos (\alpha \pi / 2)^{2}-1}\right] / C_{\alpha} R .
$$




\section{Simulation Results}

The performance of the VGC-CCIII- was tested using the the OPA860 SPICE macromodel. The DC power supply voltages were equal to $\pm 5 \mathrm{~V}$. All passive component values were selected from E48 $( \pm 2 \%)$ standard values. The $R_{A D J}$ was chosen as $270 \Omega$ (see [15]) and value of resistors $R_{i}(i=1,2$, 3 ) was set $470 \Omega$. The simulated performance characteristics of VGC-CCIII- are given in Table I. Simulated voltage gain $h$ responses between $\mathrm{Y}$ and $\mathrm{X}$ terminals are demonstrated in Fig. 4. In Fig. 4(a), the resistor $R_{h}$ has been varied from $120 \Omega$ to $1470 \Omega(h \approx\{0.25 \rightarrow 3\})$. Figure 4 (b) demonstrates wide tunability range of $h \approx\{0.1 \div 9.19\}$ by varying resistor $R_{h}$ in range $50 \Omega \rightarrow 5 \mathrm{k} \Omega$ vs. relative error. Correspondent relative error of voltage gain in range of interest is about $-3 \%$. Effect of resistors $R_{3}$ vs. $R_{h}$ on voltage gain $h$ is depicted in Fig. 5 .

\section{A. Verification of Integer-Order All-Pass Filter}

Using the VGC-CCIII- implementation from Fig. 2(c) the CM APF from Fig. 3 has also been simulated. In integer-order APF case, the passive element values were selected as $C=240 \mathrm{pF}$ and $R=1.2 \mathrm{k} \Omega$. Hence, a $-90^{\circ}$ phase shift is at pole frequency $500 \mathrm{kHz}$. Figure 6(a) gives the ideal and simulated gain and phase responses showing the tunability of the filter. The pole frequency of the circuit was varied for $f_{p_{-} \text {sim }} \approx\{0.128 ; 0.25 ; 0.497 ; 1 ; 1.449\} \mathrm{MHz}$ via voltage gain $h \approx\{0.25 ; 0.5 ; 1 ; 2 ; 3\}$, respectively. Ideal and simulated pole frequency tunability in wide range of tuning via $h$ and relative error are depicted in Fig. 6(b).

TABLE I. MAIN PERFORMANCE PARAMETERS OF THE VGC-CCIII(NOTE: $@$ @ $h \approx 1$; TPD: TOTAL POWER DISSIPATION).

\begin{tabular}{|l|l|l|l|}
\hline \multicolumn{1}{|c|}{ Parameter } & \multicolumn{1}{|c|}{ Value } & \multicolumn{1}{c|}{ Parameter } & \multicolumn{1}{c|}{ Value } \\
\hline$i_{Y} / i_{X}$ gain $(\gamma)$ & 0.961 & $i_{Z} / i_{X} f_{-3 \mathrm{~dB}}$ & $87.81 \mathrm{MHz}$ \\
\hline$v_{X} / v_{Y}$ gain $(\beta \cdot h)^{\#}$ & 0.976 & $C_{Y} \| R_{Y}$ & $4.1 \mathrm{pF} \| 48.27 \mathrm{k} \Omega$ \\
\hline$i_{Z} / i_{X}$ gain $(\eta)$ & 0.943 & $R_{X}$ & $10.5 \Omega$ \\
\hline$i_{Y} / i_{X} f_{-3 \mathrm{~dB}}$ & $84.32 \mathrm{MHz}$ & $C_{Z_{-}} \| R_{Z-}$ & $2 \mathrm{pF} \| 54 \mathrm{k} \Omega$ \\
\hline$v_{X} / v_{Y} f_{-3 \mathrm{~dB}}^{\#}$ & $87.55 \mathrm{MHz}$ & $\mathrm{TPD}$ & $302 \mathrm{~mW}$ \\
\hline
\end{tabular}

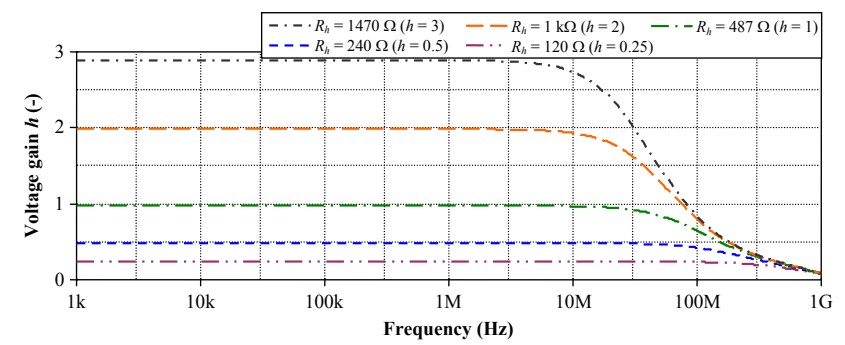

(a)

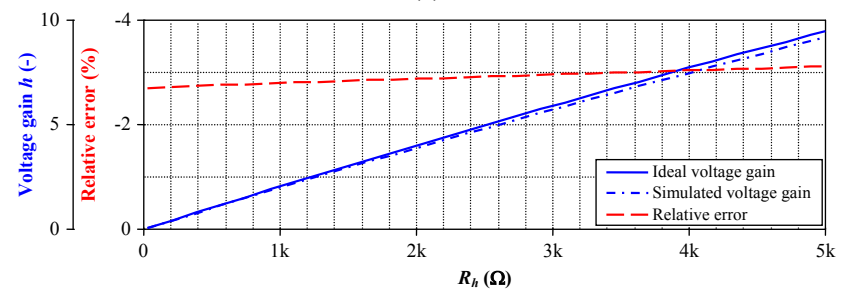

(b)

Fig. 4. (a) AC voltage transfer characteristic of the proposed minus-type VGC-CCIII-, (b) voltage gain tunability and relative error.

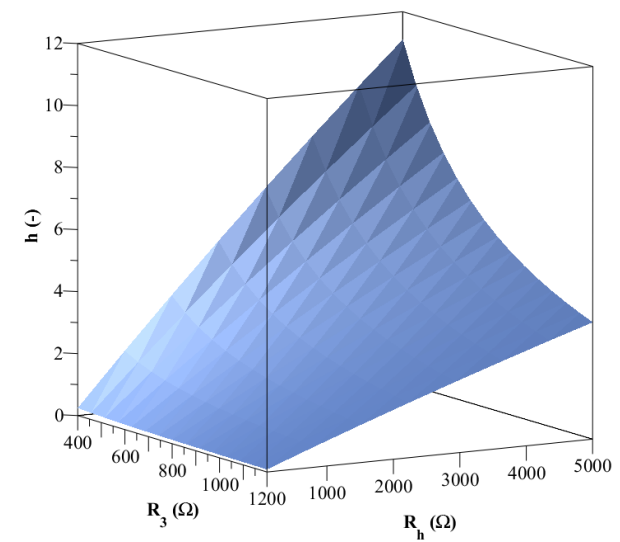

Fig. 5. Effect of resistors $R_{3}$ vs. $R_{h}$ on voltage gain $h$.

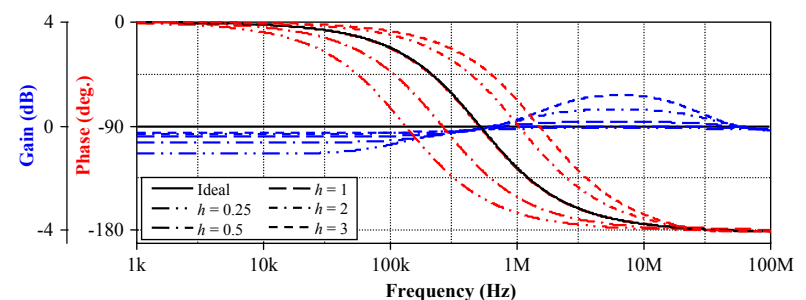

(a)

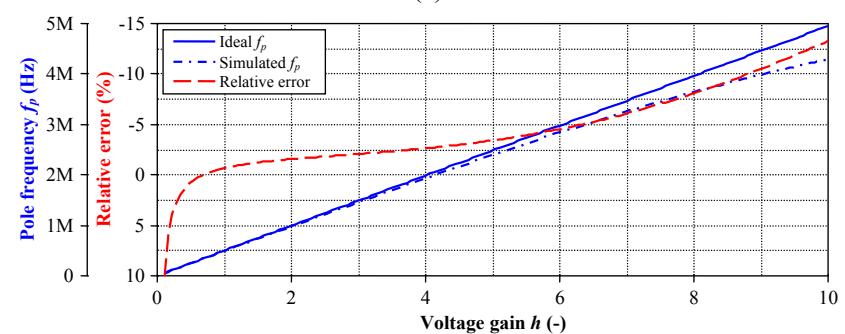

(b)

Fig. 6. Integer-order CM APF: (a) tunability of the magnitude and phase responses by voltage gain $h$, (b) pole frequency tunability and relative error.

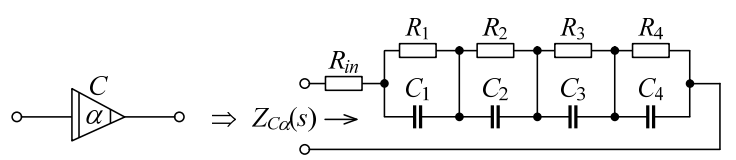

Fig. 7. RC tree realization of fractional-order capacitor $C_{\alpha}$.

TABLE II. COMPONENT VALUES USED IN FIG. 7 FOR SPICE SiMULATIONS OF $C=240 \mathrm{pF} @ 500 \mathrm{kHz}$.

\begin{tabular}{|l|c|c|c|}
\hline \multicolumn{1}{|c|}{ Values } & $\begin{array}{c}\alpha=\mathbf{0 . 3} \\
\boldsymbol{C}_{\mathbf{0 . 3}}=\mathbf{8 . 4 8} \boldsymbol{\mu} \mathbf{F} / \mathbf{s}^{\mathbf{0 . 7}}\end{array}$ & $\begin{array}{c}\boldsymbol{\alpha}=\mathbf{0 . 5} \\
\boldsymbol{C}_{\mathbf{0 . 5}}=\mathbf{4 2 5 . 3 9} \mathbf{n F} / \mathbf{s}^{\mathbf{0 . 5}}\end{array}$ & $\begin{array}{c}\boldsymbol{\alpha}=\mathbf{0 . 7} \\
\boldsymbol{C}_{\mathbf{0 . 7}}=\mathbf{2 1 . 3 5} \mathbf{~ n F} / \mathbf{s}^{\mathbf{0 . 3}}\end{array}$ \\
\hline$R_{\text {in }}(\Omega)$ & 390 & 147 & 51 \\
\hline$R_{1}(\Omega)$ & 402 & 332 & 200 \\
\hline$C_{1}(\mathrm{pF})$ & 82.5 & 120 & 270 \\
\hline$R_{2}(\Omega)$ & 422 & 510 & 422 \\
\hline$C_{2}(\mathrm{pF})$ & 470 & 430 & 620 \\
\hline$R_{3}(\Omega)$ & 681 & 1100 & 1400 \\
\hline$C_{3}(\mathrm{nF})$ & 1.15 & 0.82 & 0.82 \\
\hline$R_{4}(\mathrm{k} \Omega)$ & 2.87 & 9.53 & 33.2 \\
\hline$C_{4}(\mathrm{nF})$ & 2.4 & 1 & 0.51 \\
\hline
\end{tabular}




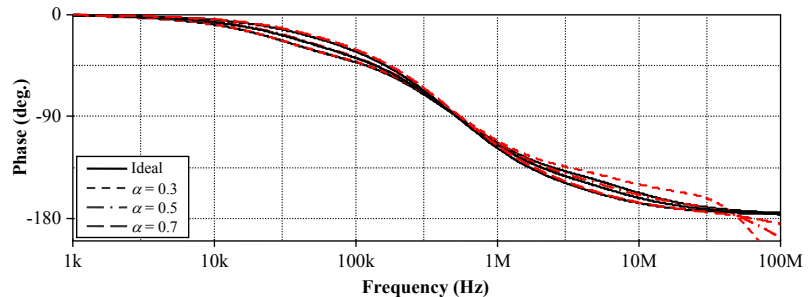

(a)

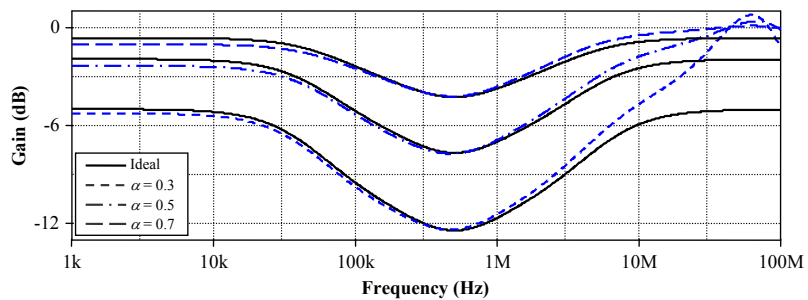

(b)

Fig. 8. Ideal and simulated (a) phase and (b) gain responses vs. frequency of CM APF of orders $\alpha=0.3 ; 0.5 ; 0.7 @ h=1$.

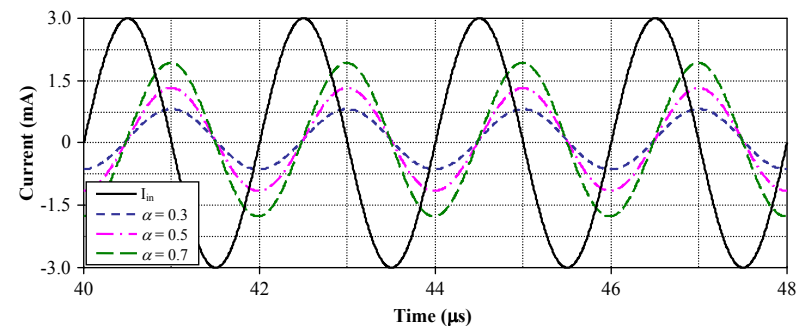

Fig. 9. Time-domain responses of CM APF of orders $\alpha=0.3 ; 0.5 ; 0.7$.

\section{B. Verification of Fractional-Order All-Pass Filter}

The performance of the proposed CM APF of orders $\alpha=0.3 ; 0.5 ; 0.7$ was further investigated and compared in SPICE software. Foster I RC tree realization from Fig. 7 approximating the fractional-order capacitor which having the same impedance $1.33 \mathrm{k} \Omega @ 500 \mathrm{kHz}$ has been employed. Using the continued fraction expansion method, computed component values are given in Table II. Ideal and simulated phase and gain responses vs. frequency of the filter @ $h=1$ are depicted in Fig. 8. As it can be seen, the phase shift in all cases is $-90^{\circ}$ and pole frequency is very close to $f_{p_{-} \text {theor }}=500 \mathrm{kHz}$. The observed deviations are mainly caused by the limitation of the employed $4^{\text {th }}$-order approximation and real behavior of used OPA860 ICs. Time-domain simulation results of the filter are shown in Fig. 9 in which a sinusoidal input current signal with $3 \mathrm{~mA}$ peak value at simulated $f_{p \text { sim }}$ was applied to the filter. The total harmonic distortion at these frequencies was found to be $\{1.18 \% ; 0.83 \% ; 0.73 \%\}$ for orders $\{0.3 ; 0.5 ; 0.7\}$, respectively.

\section{CONCLUSION}

The paper presents a new CCIII featuring with variable voltage gain, which in the implementation using the OPA860 ICs the voltage gain $h$ between $\mathrm{Y}$ to $\mathrm{X}$ terminals can be set by the ratio of two grounded resistors. The pole frequency of the CM first-order APF is successfully tuned in wide frequency range. Moreover, the performance of the CM APF was further investigated considering the fractional-orders $\alpha=0.3 ; 0.5 ; 0.7$. SPICE simulations confirmed theoretical assumptions.

\section{REFERENCES}

[1] T. Dostal, J. Pospisil, "Current and voltage conveyors-a family of threeport immitance converters," in Proc. of the Int. Symposium on Circuits and Systems (ISCAS), Roma, Italy, pp. 419-422, 1982.

[2] A. Fabre, "Third-generation current conveyor: a new helpful active element," Electron. Lett., vol. 31, no. 5, pp. 338-339, 1995.

[3] A. Piovaccari, "CMOS integrated third-generation current conveyor," Electron. Lett., vol. 31, no. 15, pp. 1228-1229, 1995.

[4] E. Yuce, B. Metin, O. Cicekoglu, "Current-mode biquadratic filters using single CCIII and minimum number of passive elements," Frequenz, vol. 58, no. 9-10, pp. 225-228, 2004.

[5] S.I. Liu, Y.Y. Yang, "Higher-order immittance function synthesis using CCIIIs," Electron. Lett., vol. 32, no. 25, pp. 2295-2296, 1996.

[6] H.-Y. Wang, Ch.-T. Lee, "Systematic synthesis of R-L and C-D immittances using single CCIII," Int. J. of Electronics, vol. 87, no. 3, pp. 293-301, 2000.

[7] H. Kuntman, M. Gulsoy, O. Cicekoglu, "Actively simulated grounded lossy inductors using third generation current conveyors," Microelectron. J., vol. 31, pp. 245-250, 2000.

[8] S. Maheshwari, I. A. Khan, "Novel first order all-pass sections using a single CCIII,” Int. J. of Electronics, vol. 88, no. 7, pp. 773-778, 2001.

[9] N. Herencsar, J. Koton, K. Vrba, O. Cicekoglu, "New current-mode allpass filter with grounded capacitor based on gain-variable CCIII," in Proc. of the 2013 AFRICON, Mauritius, pp. 1-4, 2013.

[10] H. Barthelemy, A. Fabre, "A new floating controlled resistance operating in class AB," IEEE Trans. on Circuits and Systems-I, vol. 47, no. 1, pp. 67-72, 2000.

[11] S. Minaei, O. K. Sayin, H. Kuntman, "A new CMOS electronically tunable current conveyor and its application to current-mode filters," IEEE Trans. Circuits and Systems-I, vol. 53, pp. 1448-1457, 2006.

[12] N. Herencsar, A. Lahiri, K. Vrba, J. Koton, “An electronically tunable current-mode quadrature oscillator using PCAs," Int. J. of Electronics, vol. 99, no. 5, pp. 609-621, 2012.

[13] A. De Marcellis, G. Ferri, N.C. Guerrini, G. Scotti, V. Stornelli, A. Trifiletti, "The VCG-CCII: a novel building block and its application to capacitance multiplication," Analog Integr. Circuits and Signal Process., vol. 58, no. 1, pp. 55-59, 2009.

[14] R. Sotner, J. Jerabek, N. Herencsar, Z. Hrubos, T. Dostal, K. Vrba, "Study of adjustable gains for control of oscillation frequency and oscillation condition in 3R-2C oscillator," Radioengineering, vol. 21, no. 1, pp. 392-402, 2012.

[15] Datasheet OPA860 - Wide bandwidth operational transconductance amplifier (OTA) and buffer. Texas Instruments, SBOS331C-June 2005Revised August 2008, www.ti.com.

[16] D. Biolek, V. Biolkova, "Implementation of active elements for analog signal processing by diamond transistors," in Proc. of the Int. Conf. Electronic Devices and Systems - IMAPS CS EDS 2009, Brno, Czech Republic, pp. 304-309, 2009.

[17] B. Metin, N. Herencsar, K. Vrba, "A CMOS DCCII with a grounded capacitor based cascadable all-pass filter application," Radioengineering, vol. 21, no. 2, pp. 718-724, 2012.

[18] N. Herencsar, S. Minaei, J. Koton, E. Yuce, K. Vrba, "New resistorless and electronically tunable realization of dual-output VM all-pass filter using VDIBA," Analog Integr. Circuits and Signal Process., vol. 74, no. 1, pp. 141-154, 2013.

[19] M. D. Ortigueira, "An introduction to the fractional continuous-time linear systems: the 21 st century systems," IEEE Circuits and Systems Magazine, vol. 8, no. 3, pp. 19-26, 2008.

[20] G. Tsirimokou, C. Psychalinos, A.S. Elwakil, "Emulation of a constant phase element using operational transconductance amplifiers," Analog Integr. Circuits and Signal Process., vol. 85, no. 3, pp. 413-423, 2015.

[21] S. Westerlund, L. Ekstam, "Capacitor theory," IEEE Trans. on Dielectrics and Electrical Insulation, vol. 1, no. 5, pp. 826-839, 1994.

[22] B. Maundy, A.S. Elwakil, S. Gift, "On the realization of multiphase oscillators using fractional-order allpass filters," Circuits, Systems, and Signal Processing, vol. 31, no. 1, pp. 3-17, 2012. 